\title{
Configurations of the circle of Willis: a computed tomography angiography based study on a Polish population
}

\author{
W. Klimek-Piotrowska1 ${ }^{1}$, M. Kopeć1 ${ }^{1}$ M. Kochana ${ }^{1}$, R.M. Krzyżewski ${ }^{1}$, \\ K.A. Tomaszewski ${ }^{1}$, P. Brzegowy ${ }^{2}$, J. Walocha ${ }^{1}$ \\ ${ }^{1}$ Department of Anatomy, Jagiellonian University Medical College, Krakow, Poland \\ 2Department of Radiology, Jagiellonian University Medical College, Krakow, Poland
}

[Received 18 May 2013; Accepted 14 July 2013]

\begin{abstract}
The aim of the study was to investigate the distribution of the circle of Willis variants in Polish population by means of computed tomography angiography (CTA). The results were then analysed and compared with another study that used similar methods but that was carried out on an ethnically distinct population. Patients presenting with intracranial pathology were excluded from the initial study population. In total, 250 CTA belonging to 129 female and 121 male patients were reviewed. A modified classification system of the circle was proposed, which took into consideration the anterior and the posterior aspects of the circle individually. The typical variant of Willis's circle occurred in $16.80 \%$ of cases. The anterior and the posterior portions of the circle were normal in $47.20 \%$ and $26.80 \%$ of the patients respectively. As for the anterior part, lack of the anterior communicating artery was the most frequent abnormality (22.80\%). Bilateral absence of posterior communicating arteries was the most common anomaly in the posterior part of the circle (29.20\%). This type of anomaly was also the most common, when taking into consideration the entire circle (12.00\%). There were statistically significant differences between the age groups and genders when considering the occurrence of an incomplete circle. Overall, a substantial proportion of patients manifested clinically important variants that were incapable of providing collateral circulation. Comparison with other imaging-based and cadaveric studies revealed noticeable differences, that may have resulted from the variable technical features of other studies or other factors such as the ethnical origins of the studied populations. (Folia Morphol 2013; 72, 4: 293-299)
\end{abstract}

Key words: cerebral arterial circle, computed tomography angiography, anatomic variations

\section{INTRODUCTION}

The circle of Willis (CW) facilitates the appropriate distribution of blood and nourishment to the brain tissues. In its typical configuration, the CW allows for effective collateral circulation when needed. Many factors play a role in the development of the structures of the CW, including one's genetic background, the haemodynamic and environmental conditions of prenatal and postnatal growth, the development of the brain, and contingent co-morbidities [1, 11, $17,18]$. As such, it is rather unsurprising that there is a large variety of configurations of the CW among both healthy and unhealthy populations of different ethnic groups $[4,11]$.

Variants that are characterised by impaired collateral flow, carry with them an increased risk of ischaemic 
events $[10,14,15,19]$. These incidents may result from disorders such as occlusive disease $[8,14]$ or from surgical procedures, for instance aortic dissection (intraoperative ischaemia) [15]. Additionally, the role of the CW in migraine [3] and aneurysm development [2] has been widely studied. In the light of the CW's key function in pathological processes, the need for thorough investigation of the appearance of different variants of the circle is understandable.

Since first described by Willis, the cerebral arterial circle has been the subject of multiple investigations. Cadaveric studies are still widely performed $[4,5,11,15]$ and several imaging methods, including transcranial colour-coded duplex ultrasonography (TCCD) [9], 3-dimensional time-of-flight magnetic resonance angiography (3D-TOF-MRA) [2, 3, 14], and computed tomography angiography (CTA) $[2,7,13,16]$ have been used to examine the circle. These imaging techniques have allowed for the precise depiction of the clinically relevant collaterals of the circle by assessing the patency of blood vessels that determine the circle's physiological completeness. A limitation of some imaging-based studies is that the study population consists of patients suffering from cerebrovascular disease. Even though many cadaveric studies have focused on the structure of the CW, some authors still consider the clinical relevance of post-mortal examination insufficient $[1,13]$.

In this study, CTA was used to explore the distribution of the various combinations of the $\mathrm{CW}$ in a healthy Polish population. To the authors' best knowledge, no CTA-based research pertaining to the variants of the cerebral arterial circle have been performed on a Polish population. Additionally, none of the other published studies have compared the results of 2 CTA-based studies, as done in this paper.

\section{MATERIALS AND METHODS}

\section{Patient population}

The initial patient population consisted of patients who underwent CTA at the Department of Radiology of the Jagiellonian University Medical College during the period from July 2008 to October 2011. Patients diagnosed with arterial aneurysms, arterio-venous malformations, subarachnoid or intra-cerebral haemorrhages, intracranial neoplasms, transient ischaemic attacks, ischaemic strokes, migraines, severe trauma, as well as those with non-diagnostic quality images, were excluded from the study.

\section{Imaging}

All participants underwent cranial CTA using a multi-row computed tomography scanner (Somatom Sensation 16; Siemens AG, Germany) with the following parameters of the study: exposure factors $120 \mathrm{kV}, 74 \mathrm{~mA}, 120 \mathrm{mAs}$; rotation time -0.75 s; slice thickness $-3 \mathrm{~mm}$; pitch -1.5 . The data obtained was sent to a workstation equipped with software for further analysis. Maximum-intensity projection reconstructions in 3 perpendicular planes - coronal, sagittal and axial - were acquired.

\section{Data evaluation}

Evaluation of all CTA was performed by three of the authors. Angiograms were carefully studied and component vessels were examined in order to reveal any abnormalities in their size, origin, course and number. The results of the examination were saved on an evaluation form together with a sketch. In cases where the evaluation was problematic due to an inferior quality image or extraordinary abnormalities, the observers consulted each other in regard to the evaluation.

The term 'typical CW' was used to describe a cerebral arterial circle in which all component vessels were present and not hypoplastic. Anterior and posterior parts of the circle were referred to as typical accordingly. The circle was considered to be complete when all the segments were depicted on CTA. In comparison, closed $\mathrm{CW}$ was defined as one displaying continuity of blood flow, regardless of presence of all components of CW.

\section{Statistical analysis}

After determining the basic elements of descriptive statistics (mean, standard deviation, percentage distribution), the Pearson $\chi^{2}$ test was used to compare the collected data with those obtained by previous studies. Where necessary, Yates correction for continuity, Cramér's V, and the Pearson contingency coefficient were applied. All calculations were done using Statistica v. 10 (Stat Soft Poland).

\section{RESULTS}

250 subjects - 129 female and 121 male, underwent evaluation of the cerebral arterial circle. The age characteristics of the study population are detailed in Table 1. CTA did not reveal any abnormalities in $73.20 \%$ of the cases. The remaining subjects manifested a variety of pathological findings (Fig. 1).

To address the wide diversity of CW configurations, our predecessors have developed a classifica- 
Table 1. Age characteristics of the patient population

\begin{tabular}{lccccc}
\hline & Number & Minimal age & Maximal age & Mean & Standard deviation \\
\hline All subjects & 250 & 15 & 89 & 45.55 & 18.33 \\
Male & 121 & 15 & 86 & 48.03 & 17.02 \\
Female & 129 & 16 & 89 & 43.22 & 19.26 \\
\hline
\end{tabular}

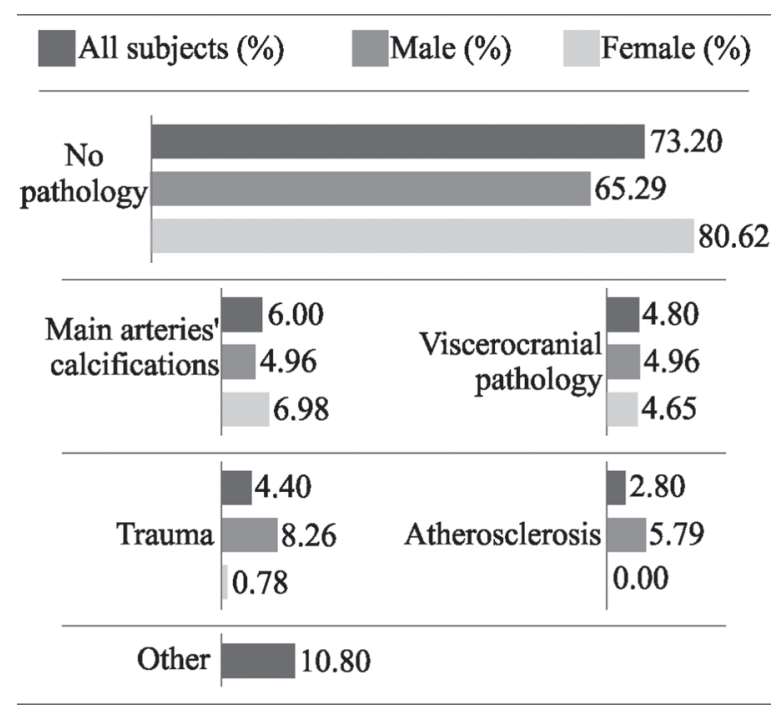

Figure 1. Percentage distribution of various computed tomography angiography findings.

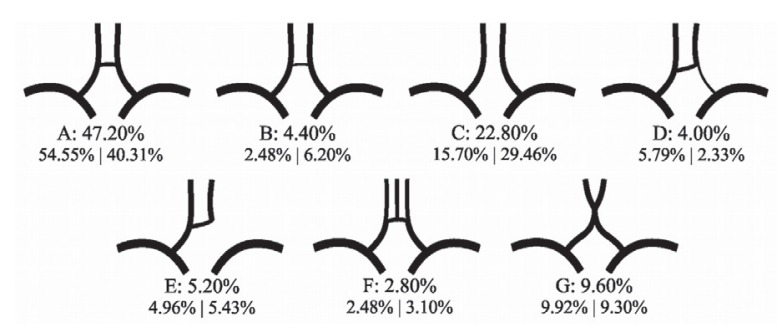

Figure 2. Percentage distribution of different types distinguished in the anterior portion of the circle of Willis in all the subjects, male/female.

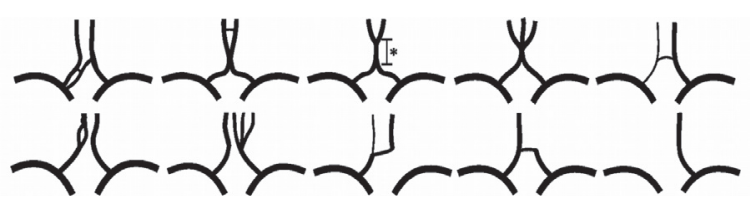

Figure 3. Ten unclassified variants of the anterior portion of the circle of Willis; *this pattern differs from type $\mathrm{G}$ in length of the common part of anterior cerebral arteries.

tion system based on dividing the CW into anterior and posterior parts, and our assessment adopts this approach [13]. The subgroups used in this paper differ from those formerly issued since the subgroups used in our paper were devised on the basis of the results obtained during our research.

\section{Anterior portion of the circle}

Seven different types of the anterior part of the $\mathrm{CW}$ were found in the study (Fig. 2). The most common configuration among the examined subjects was type A (typical), observed in $47.20 \%$ of the cases. Lack of the anterior communicating artery (type C) and accretion of the anterior cerebral arteries (ACAs) (type G) were also frequent variations, occurring in $22.80 \%$ and $9.60 \%$ of the cases, respectively.

We encountered $10(4.00 \%)$ patients in whom anterior $\mathrm{CW}$ variations differed so distinctly from more common patterns, that they could not be classified within the established 7 types. They are illustrated individually on Figure 3. Five cases belonged to male subjects and 5 belonged to female subjects. Of these 10 cases, 2 cases pertained to additional arteries, 2 cases pertained to unusually joined ACAs, while 2 cases involved arteries that had an unusual course. The remaining cases were characterised by the presence of hypoplastic vessels, or the complete absence of 2 or more segments.

The anterior portion of the $\mathrm{CW}$ was noted to be open in $29.60 \%$ of the cases. As such, collateral blood flow was impaired in these subjects. Females were found to have a significantly higher rate of an open anterior part of the circle when compared to males (35.66\% vs. $23.14 \%, p<0.05)$. There were no relevant differences in the incidence of variants between male and female subjects when testing conjointly. Nevertheless, testing individually for each pattern revealed that the typical variant was more frequent in men $(19.83 \%$ vs. $13.95 \%, p<0.05)$, while the one lacking the anterior communicating artery (AComA) appeared more often in women $(29.46 \%$ vs. $15.70 \%, p<0.01)$. No age-related differences were observed in regard to the prevalence of types or in regard to impaired collateral function.

\section{Posterior portion of the circle}

In the posterior part of the circle, 14 various types were distinguished (Fig. 4). The absence of both po- 


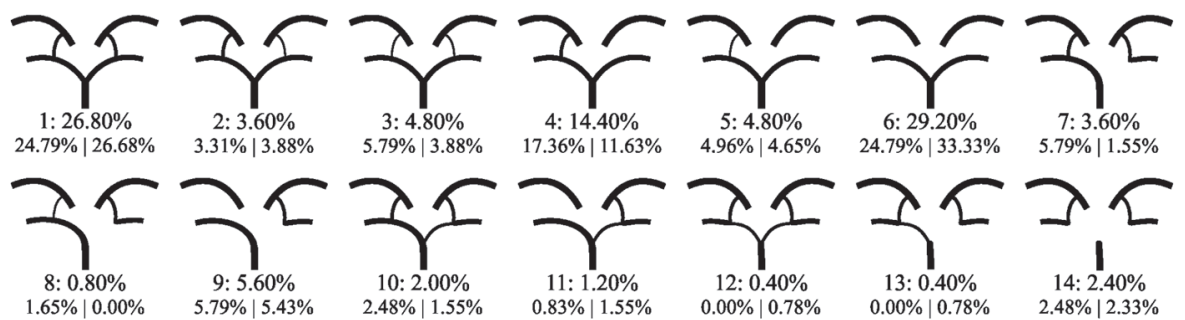

Figure 4. Percentage distribution of various types distinguished in the posterior portion of the circle of Willis in all the subjects, male/female.

Table 2. Incidence of segment absence and hypoplasia in the anterior and posterior portion of the circle

\begin{tabular}{|c|c|c|c|c|c|c|}
\hline & & \multicolumn{3}{|c|}{ A. Anterior } & \multicolumn{2}{|c|}{ B. Posterior } \\
\hline & & AComA & & $\mathrm{A} 1$ & P1 & PComA \\
\hline \multirow{6}{*}{ Hypoplastic } & Men & $3.30 \%$ & UL & $6.61 \%$ & $3.31 \%$ & $9.92 \%$ \\
\hline & & & $\mathrm{BL}$ & - & - & $5.79 \%$ \\
\hline & Women & $6.20 \%$ & UL & $2.33 \%$ & $3.88 \%$ & $8.53 \%$ \\
\hline & & & $B L$ & - & $0.80 \%$ & $3.88 \%$ \\
\hline & All & $4.80 \%$ & UL & $4.40 \%$ & $3.60 \%$ & $9.20 \%$ \\
\hline & subjects & & $\mathrm{BL}$ & - & $0.40 \%$ & $4.80 \%$ \\
\hline \multirow{6}{*}{ Absent } & Men & $27.27 \%$ & UL & $6.61 \%$ & $13.22 \%$ & $28.93 \%$ \\
\hline & & & $\mathrm{BL}$ & - & $2.48 \%$ & $24.79 \%$ \\
\hline & Women & $41.56 \%$ & UL & $5.43 \%$ & $7.75 \%$ & $23.26 \%$ \\
\hline & & & $\mathrm{BL}$ & - & $2.33 \%$ & $33.33 \%$ \\
\hline & All subjects & $34.80 \%$ & UL & $6.00 \%$ & $10.40 \%$ & $26.00 \%$ \\
\hline & & & $\mathrm{BL}$ & - & $2.40 \%$ & $29.20 \%$ \\
\hline Other & & $0.40 \%$ & & & & \\
\hline AComA & Men & $2.48 \%$ & & & & \\
\hline with additional & Women & $3.10 \%$ & & & & \\
\hline $\mathrm{ACA}^{\mathrm{a}}$ & All subjects & $2.80 \%$ & & & & \\
\hline
\end{tabular}

aAlso known as a median artery of corpus callosum; ACA — anterior cerebral arteries; AComA — anterior communicating artery; A1 — pre-communicating part of anterior cerebral artery; $\mathrm{P} 1$ — pre-communicating part of posterior cerebral artery; $\mathrm{PComA}$ — posterior communicating artery; UL — unilaterally; $\mathrm{BL}$ — bilaterally

sterior communicating arteries (type 6) was the most common variant, appearing in $29.20 \%$ of the cases. The typical pattern (type 1) had a similar prevalence and occurred in $26.80 \%$ of the subjects. Apart from 2 other configurations (type 4, type 9), the remaining types occurred in no more than $5.00 \%$ of the cases each.

The posterior part of the circle was complete and closed in $37.60 \%$ of the cases, providing collateral blood flow. Conversely, $62.40 \%$ of the subjects were found to have the posterior component deficient in 1 or more segments, forming an open posterior part. A foetal type posterior circle (FTP) appeared in $16.40 \%$ of the cases, including both full (types 7, 8, 9, 13) and partial FTP (types 10-14). There were no gender-related differences in the incidence of segment absence or hypoplasia in the posterior portion of the circle. Patients above 46 years of age (median) had the incomplete posterior portion of CW more often (68.55\% vs. $56.35 \%$; $p<0.05$ ).

\section{Entire circle}

The typical variant was the most common combined type of CW in this study, appearing in $16.80 \%$ of the cases. We observed that $12.00 \%$ of the subjects presented with typical anterior part of the circle combined with bilateral absence of the posterior communicating arteries (PComAs). These 2 patterns occurred just as frequently in both genders. Looking at the CW as a whole, lack of all communicating arteries (type C-6) and the isolated absence of the AComA (type C-1) were also frequent, appearing in $7.20 \%$ and $5.60 \%$ of the cases, respectively. Female subjects were noted to present with a markedly higher prevalence of these variants: $10.08 \%$ vs. $4.13 \%$ for type C- 6 and $9.30 \%$ vs. $1.65 \%$ for type C-1. The posterior portion of the circle was more often open than the anterior part $(62.40 \%$ and $29.60 \%$, respectively; $p<0.001)$. The entire circle was open in $69.20 \%$ of the subjects, 
and more frequently in subjects of 46 or more years of age $(p<0.05)$.

\section{Segments of the circle}

The incidence of segment absence and hypoplasia in the circle is shown in Table 2. Lack of AComA was seen in $41.56 \%$ of female and $27.27 \%$ of male patients. This data does not correlate with the prevalence of type $C$ shown on Figure 2, owing to the fact that accretion of the ACAs (type G) and 5 unclassified configurations were also considered as a deficiency of the AComA. Bilateral absence of PComAs was observed in 33.33\% of women and $24.79 \%$ of men, while the trend for the unilateral lack of this segment was reversed - seen in $28.93 \%$ of male and $23.26 \%$ of female patients.

\section{DISCUSSION}

Many authors have hypothesised that there are considerable differences in the prevalence of various configurations of the $\mathrm{CW}$ in various ethnic groups $[4,11,13]$. Conclusions based on the comparison of data acquired using different methods seem to be questionable. This is due to the fact that each particular evaluation technique has different characteristics and limitations $[5,6,9]$. Furthermore, it should be noted that a number of previously published studies were carried out on patients with cerebrovascular disorders $[3,6,8,11]$.

In this paper, we decided to compare our results to those reported in another study [13] that has several similarities to our own. First of all, it was a CTA-based study, although it used a Chinese population [13]. The study in question had similar inclusion criteria, so as to exclude patients with disorders of the cerebral vessels. Lastly, the authors of the aforementioned study divided the CW into anterior and posterior parts, as in our study.

The incidence of particular variants of the anterior part of the CW differed significantly between both studies, as shown in Table 3A. The typical pattern was over 1.5 times more frequent in the study conducted by Li et al. [13], whereas lack of AComA was observed approximately twice as often in the present study. $20.80 \%$ of the cases noted in the present study did not have a counterpart in the aforementioned study. Variants with an additional artery originating from the AComA (type F) and with accretion of the ACAs (type G) were included in these cases. It is the authors' opinion that these variants provided collateral blood flow to this part of the circle. A hypoplastic AComA or precommunicating segment of the ACA (A1) may be insufficient to provide collateral circulation. If so, the occurrence of
Table 3. Comparison of the obtained results with a similar computed tomography angiography-based study by Li et al. [14]

\begin{tabular}{|c|c|c|c|}
\hline \multicolumn{3}{|c|}{ A. Anterior part } & \multirow[b]{3}{*}{$\mathbf{P}$} \\
\hline & Current study & Li et al. [14] & \\
\hline Type & Frequency & Frequency & \\
\hline$A$ & $47.20 \%$ & $76.25 \%$ & $<0.0001^{*}$ \\
\hline B & $4.40 \%$ & $x$ & \\
\hline C & $22.80 \%$ & $9.38 \%$ & $0.0261^{*}$ \\
\hline D & $4.00 \%$ & $10.00 \%$ & $0.0008^{*}$ \\
\hline$E$ & $5.20 \%$ & $1.88 \%$ & $0.1523^{*}$ \\
\hline $\mathrm{F}$ & $2.80 \%$ & $x$ & \\
\hline G & $9.60 \%$ & $x$ & \\
\hline \multirow[t]{2}{*}{ S } & $4.00 \%$ & $x$ & \\
\hline & $x$ & $2.50 \%$ & \\
\hline \multicolumn{3}{|c|}{ B. Posterior part } & \multirow[b]{3}{*}{$\mathbf{P}$} \\
\hline & Current study & Li et al. [14] & \\
\hline Type & Frequency & Frequency & \\
\hline 1 & $26.80 \%$ & $\begin{array}{cc}21.88 \% \%^{a} & 17.50 \% \\
& 4.38 \%\end{array}$ & 0.3139 \\
\hline 2 & $3.60 \%$ & $9.38 \%$ & $0.0267^{*}$ \\
\hline 3 & $4.80 \%$ & $13.75 \%$ & $0.0025^{*}$ \\
\hline 4 & $14.40 \%$ & $10.00 \%$ & 0.2486 \\
\hline 6 & $29.20 \%$ & $33.13 \%$ & 0.4657 \\
\hline 7 & $3.60 \%$ & $1.25 \%$ & 0.2613 \\
\hline 10 & $2.00 \%$ & $6.88 \%$ & $0.0259^{*}$ \\
\hline 12 & $0.40 \%$ & $2.50 \%$ & 0.1531 \\
\hline \multirow[t]{2}{*}{$\begin{array}{c}5,8,9,11 \\
13,14\end{array}$} & $15.20 \%$ & $x$ & \\
\hline & $x$ & $1.25 \%$ & \\
\hline
\end{tabular}

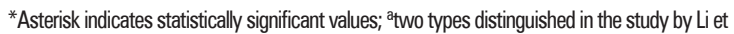
al. [14] corresponded to type 1 of the posterior part of the circle, thus they were compared jointly

variants that did not secure brain tissue perfusion in occlusive disease is noticeably higher in the present study.

Both studied populations were found to have a similar occurrence of the most frequent variants of the posterior part of the CW: bilateral absence of the PComAs, the typical configuration, and unilateral lack of PComA, respectively (Table $3 \mathrm{~B}$ ). In comparison, the frequency of unilateral and bilateral PComAs hypoplasia differed significantly, occurring almost 3 times more often in the Chinese study [13]. Some of the types presented in the current study were not distinguished by Li et al. [13], and were mostly variants of the partial and full foetal type configuration. FTP appeared more often in the current study, especially full FTP, which was 10 times more frequent than in the 
Table 4. Comparison of the obtained results with data from literature based on imaging studies

\begin{tabular}{lcccc}
\hline Study & Current & $\begin{array}{c}\text { Li } \\
\text { et al., 2011 [14] }\end{array}$ & $\begin{array}{c}\text { El-Barhoun } \\
\text { et al., 2009 [7] }\end{array}$ & $\begin{array}{c}\text { Krabbe-Hartkamp } \\
\text { et al., 1998 [13] }\end{array}$ \\
\hline Method & CTA & CTA & MRI & MRI \\
Number of subjects & 250 & 160 & 171 & 150 \\
Anterior part closed & $70.40 \%$ & $79.00 \%$ & $83.00 \%$ & $74.00 \%$ \\
Posterior part closed & $37.60 \%$ & $31.00 \%$ & $52,60 \%$ & $52.00 \%$ \\
Entire circle closed & $30.80 \%$ & $27.00 \%$ & $44.00 \%$ & $42.00 \%$ \\
Most often type & BPComAs absence & BPComAs absence & BPComAs absence & BPComAs absence \\
\hline
\end{tabular}

CTA — computed tomography angiography; $\mathrm{MRI}$ — magnetic resonance imaging; BPComA — bilateral posterior communicating artery

Table 5. Comparison of the obtained results with data from literature based on cadaveric studies

\begin{tabular}{lcccc}
\hline Study & Current & Kapoor et al., & $\begin{array}{c}\text { Eftekhar et al., } \\
\text { 2006 [6] }\end{array}$ & $\begin{array}{c}\text { De Silva et al., } \\
\text { 2011 [4] }\end{array}$ \\
\hline Method & 2008 [12] & Autopsy & Autopsy & Autopsy \\
Number of subjects & CTA & 100 & 102 & 225 \\
Entire circle closed & 250 & $96.80 \%$ & $89.00 \%$ & $100.00 \%$ \\
Most often type & $30.80 \%$ & BPComAs hypoplasia & BPComAs hypoplasia & BPComAs hypoplasia \\
\hline
\end{tabular}

CTA — computed tomography angiography; BPComA — bilateral posterior communicating artery

Chinese study [13]. The posterior portion of the circle was complete and therefore able to provide collateral blood flow with similar frequency in both groups.

Compared to other studies carried out using imaging techniques, our results showed a considerably lower incidence of the typical anterior and posterior parts of the CW: $47.20 \%$ vs. $69.90 \%$ and $26.80 \%$ vs. $44.40 \%$, respectively [6]. While the lack of AComA was twice as frequent as in a MRA-based study on an Australian population, accretion of the ACAs was present almost equally often [6]. The prevalence of a closed anterior circle ranged from $74.00 \%$ to $83.00 \%$ (Table 4) in the studies cited. The posterior component of the circle was incomplete in approximately half of the subjects in two MRA-based studies [6, 14] and one TCCD-based [9] study. The present study, as well as the CTA-based research carried out by $\mathrm{Li}$ et al. [13], showed a noticeably higher occurrence of an open posterior part of the CW (Table 4). Bilateral lack of PComAs was the most frequent posterior variant published in the literature [6, 12-14], although its prevalence varied. This variant was reported in about $30.00 \%$ of the subjects undergoing CTA examinations, which was twice as often as in the aforementioned MRA and TCCD studies [6,9]. The incidence of FTP has been reported as ranging from $11.20 \%$ to $18.00 \%$ in different studies $[6,14]$, whereas in the present study it was found to occur in $16.40 \%$ of the cases. The entire circle was open in more than half of the subjects when taking into account all the cited studies. The higher prevalence of an open anterior part of the circle in the female subjects remains unverified. The frequency of an open CW was confirmed to increase with age in one of the cited papers [6].

Cadaveric examinations have yielded a range of inconsistent results $[4,5,11]$ that do not correspond with imaging studies. The prevalence of the typical pattern of the $\mathrm{CW}$ has been reported to range from $14.00 \%$ to $45.20 \%$ in cadaveric examinations $[4,11]$, in contrast to imaging studies where it has been observed in $22.29 \%$ to $45.76 \%$ [6, 9] of the subjects. As shown in Table 5, the entire circle was reported to be open rather rarely - in $3.20 \%$ to $11.00 \%$ of the subjects $[5,11]$. It was also found to be always closed [4] in one study. The absence of the AComA was noted in no more than $2 \%$ of the patients, 5 to 10 times less than what has been reported in imaging studies $[6,13]$. The most frequent type of posterior circle observed in autopsy studies was bilateral PComAs hypoplasia (Table 5), present in about a quarter of the cases $[4,5]$.

The reason for the differences noted above is that the luminal diameters of arteries are known to vary between cadaveric and imaging studies [4, 5, 13]. Transmural pressure distends arterial vessels in the presence of blood flow and adequate blood pressure. During post-mortem evaluation, blood vessels collapse. Preserving techniques may also influence the caliber of vessels $[4,13]$. Additionally, external mea- 
surements do not allow for the evaluation of vessel patency. A considerable proportion of hypoplastic arteries observed during autopsy do not facilitate perfusion. As noted above, CW abnormalities most commonly involved both PComAs that were either hypoplastic (autopsy) or absent (imaging).

The studies confirm that CTA is an accurate imaging technique for the evaluation of the structures of the CW $[7,16]$. It is highly sensitive and specific, except when it comes to detecting hypoplastic segments, where it has a rather low sensitivity [7]. Additionally, CTA allows for exact assessment of the presence of the foetal configuration of the posterior part of the circle [16]. The main limitations of this study correspond to those of imaging techniques in general and particularly to limitations of CTA. As shown, especially for arteries of small diameter such as AComA and PComAs, there are considerable discrepancies between imaging and cadaveric studies.

\section{CONCLUSIONS}

This paper shows that the prevalence of the normal CW constitutes a minority of cases in a healthy population $(16.80 \%)$. Interestingly, $69.20 \%$ of the patients were noted to have an incomplete cerebral arterial circle that was unable to provide collateral blood flow. Female subjects and patients over 46 years of age were noted to have a higher occurrence of such deficient variants of the CW. Additionally, female subjects had a significantly higher proportion of an open anterior part of CW. The results of our research were compared with other studies conducted, using imaging techniques and revealed noticeable differences. Unfortunately, comparing studies proved to be challenging and was impeded by dissimilarities in the study population characteristics, the technical parameters of imaging used, and how various types of $\mathrm{CW}$ variants were classified. Therefore, it is suggested that future studies concerning the CW should be carried out using more comparable methods.

\section{ACKNOWLEDGEMENTS}

For the access to a database of radiologic examinations: Andrzej Urbanik, Head of the Department of Radiology, Jagiellonian University Medical College, Krakow, Poland.

\section{REFERENCES}

1. Ardakani SH, Dadmehr M, Nejat F (2008) The cerebral arterial circle (Circulus Arteriosus Cerebri) an anatomical study in fetus and infant samples. Pediatr Neurosurg, 44: 388-392.
2. Bor ASE, Velthuis BK, Majoie CB, Rinkel GJ (2008) Configuration of intracranial arteries and development of aneurysms. Neurol, 70: 700-705.

3. Bugnicourt JM, Garcia PY, Peltier J, Bonnaire B, Picard C, Godefroy $O$ (2009) Posterior circle of Willis: a risk factor for migraine? Headache, 49: 879-886.

4. De Silva KRD, Silva R, Amaratunga D, Gunasekera WS, Jayesekera RW (2011) Types of the cerebral arterial circle (circle of Willis) in a Sri Lankan Population. BMC Neurology, 11: 5.

5. Eftekhar B, Dadmehr M, Ansari S, Ghodsi M, Nazparvar B, Ketabchi E (2006) Are the distributions of variations of circle of Willis different in different populations? Results of an anatomical study and review of literature. BMC Neurology, 6: 22.

6. El-Barhoun EN, Gledhill SR, Pitman AG (2009) Circle of Willis artery diameters on MR angiography: An Australian reference database. JMIRO J Med Imaging and Radiat Oncology, 53: 248-260.

7. Han A, Yoon DY, Chang SK, Lim KJ, Cho BM, Shin YC, Kim SS, Kim KH. (2011) Accuracy of CT angiography in the assessment of the circle of Willis: comparison of volume-rendered images and digital subtraction angiography. Acta Radiol, 52: 889-893.

8. Hoksbergen AWJ, Legemate DA, Csiba L Csáti G, Síró P, Fülesdi B (2003) Absent collateral function of the circle of willis as risk factor for ischemic stroke. Cerebrovasc Dis, 16: 191-198.

9. Hoksbergen AWJ, Legemate DA, Ubbink DT, Jacobs MJ (2000) Collateral variations in circle of Willis in atherosclerotic population assessed by means of transcranial color-coded duplex ultrasonography. Stroke, 31: 1656-1660.

10. Kayembe KNT, Sasahara M, Hazama F (1984) Cerebral aneurysms and variations in the circle of Willis. Stroke, 15: 846-850.

11. Kapoor K, Singh B, Dewan LIJ (2008) Variations in the configuration of the circle of Willis. Anatomical Science International, 83: 96-106.

12. Krabbe-Hartkamp MJ, van der Grond J, de Leeuw FE de Groot JC, Algra A, Hillen B, Breteler MM, Mali WP (1998) Circle of Willis: morphologic variation on three-dimensional time-of-flight MR angiograms. Radiology, 207: 103-111.

13. Li Q, Li J, Lv F, Li K, Luo T, Xie P (2011) A multidetector CT angiography study of variations in the circle of Willis in a Chinese population. J Clin Neurosci, 18: 379-383.

14. Macchi C, Lova RM, Miniati B, Gulisano M, Pratesi C, Conti AA, Gensini GF. (2002) The circle of Willis in healthy older persons. J Cardiovasc Surg (Torino), 43: 887-890.

15. Papantchev V, Hristov S, Todorova D, Naydenov E, Paloff A, Nikolov D, Tschirkov A, Ovtscharoff W (2007) Some variations of the circle of Willis, important for cerebral protection in aortic surgery: a study in Eastern Europeans. Eur J Cardiothorac Surg, 31: 982-989.

16. Van Der Lugt A, Buter TC, Govaere F (2004) Accuracy of CT angiography in the assessment of a fetal origin of the posterior cerebral artery. Eur Radiol, 14: 1627-1633.

17. Van Overbeeke JJ, Hillen B, Tulleken CAF (1991) A comparative study of the circle of Willis in fetal and adult life. The configuration of the posterior bifurcation of the posterior communicating artery. J Anat, 176: 45-54.

18. Van Raamt AF, Mali WPTM, van Laar PJ, van der Graaf Y (2006) The fetal variant of the circle of Willis and its influence on the cerebral collateral circulation. Cerebrovasc Dis, 22: 217-224.

19. Yu-Ming C, Chih-Yang L, Po-Jung P, Ching-Po L (2008) Posterior communicating artery hypoplasia as a risk factor for acute ischemic stroke in the absence of carotid artery occlusion. J Clin Neurosci, 15: 1376-1381. 